\title{
The role of bacterial biofilm in persistent infections and control strategies
}

\author{
Li Chen*, Yu-mei Wen
}

Key Laboratory of Medical Molecular Virology of Ministry of Education and Ministry of Public Health, Shanghai Medical School of Fudan University, Shanghai 200032, China

Bacterial biofilms can be viewed as a specific type of persistent bacterial infection. After initial invasion, microbes can attach to living and non-living surfaces, such as prosthetics and indwelling medical devices, and form a biofilm composed of extracellular polysaccharides, proteins, and other components. In hosts, biofilm formation may trigger drug resistance and inflammation, resulting in persistent infections. The clinical aspects of biofilm formation and leading strategies for biofilm inhibitors will be discussed in this mini-review.

Keywords: biofilm; persistent infection; 3 A remedies

International Journal of Oral Science (2011) 3: 66-73. doi: 10.4248/IJOS 11022

\section{Introduction}

Persistent infections are a global challenge for human beings, claiming millions of lives every year and demanding huge medical and social resources. The development of persistent infections has been exemplified as a game of "Cat \& Mouse" in which the host tries to eliminate a pathogen while the pathogen tries to survive in the host. One common survival strategy employed by bacteria pathogens is to form a biofilm, an amorphous and dynamic structure that is not only resistant to antibiotics, but also resistant to host immune clearance. In bacterial infections affecting internal organs, such as Pseudomonas aeruginosa ( $P$. aeruginosa) in cystic fibrosis pneumonia [1], Escherichia coli (E. coli) in urinary tract infections [2], and Mycobacterium tuberculosis (M. tuberculosis) in human tuberculosis [3] biofilms provide an important reservoir of cells that can repopulate colonized sites. Biofilms are also responsible for persistent Streptococcus mutans (S. mutans) infections on tooth surfaces. The $S$. mutans level rebounds in days, even after a combination

*Correspondence: Li Chen

Tel: 8621 54237236; Fax: 862164174578

E-mail: lichen_bk@fudan.edu.cn

Received 26 February 2011; Accepted 13 March 2011 of professional mechanical tooth cleaning and topical antimicrobial treatments. In addition, most nosocomial infections are persistent biofilm infections [4-5]. It is estimated that, in developed countries, over $60 \%$ of treated infectious conditions are caused by biofilm formation.

As a correlation between biofilm formation and bacterial persistence has been established [6], the possibility of using drugs targeting biofilm formation in combination with the current antibiotics is emerging as a potential therapeutic approach for this type of bacterial persistent infection. Several anti-biofilm and/or biofilm control strategies, such as anti-adhesion, quorum sensing disruption and selective targeted anti-microbial peptides, have been recently developed. These strategies will be discussed. The readers interested in the structure and function of biofilms and biofilm diseases are recommended to read the reviews by Dr. Costerton and his colleagues [4-5].

\section{Molecular regulation of biofilm formation}

\section{Biofilm formation is a two-stage process}

Biofilms are surface bacterial aggregates encased in a synthesized hydrated matrix [5]. In general, biofilm forma- 
tion is a two-stage process that begins with the adherence of bacteria to a substrate surface (Adhesion Stage), and continues by proliferation and differentiation of the attached cells (Maturation Stage). From a molecular biology point of view, these two stages are mainly controlled by surface adhesins and cell-to-cell communication signaling pathways respectively.

\section{Adhesins are key regulators of the adhesion stage}

Pathogens colonize different sites in the human body because they express multiple adhesins. These are usually proteins that recognize specific receptors expressed at various sites of the host. Bacteria also produce different types of polysaccharides that are specifically designed to form the structural components of the biofilm. The expression of adhesins seems to be regulated by a variety of inputs. For Staphylococcus epidermidis (S. epidermidis), initial adhesion to the naked or coated polymer surface is mediated by polysaccharide adhesin (PS/A) [7-8]. The expression of PS/A is controlled by the intercellular adhesion operon (Ica). In S. mutans, the adhesin SpaP (PAc) is critical for $S$. mutans's adhesion to tooth surfaces and the process is further enhanced by sucrose or pre-existing biofilms [9].

Although $S$. mutans is normally known as an oral bacterium, and the etiological agent for dental caries, it is also implicated in bacteremia and infective endocarditis. Apparently, different adhesins may participate in the adhesion of S. mutans on different tissues. Based on the chemical composition of serotype-specific polysaccharides, S. mutans can be classified into serotypes c, e, f and k with approximately $70 \%-80 \%$ of strains found in the oral cavity classified as serotype c, followed by e $(\sim 20 \%)$, and $\mathrm{f}$ and $\mathrm{k}$ (less than $5 \%$ each). Serotype $\mathrm{k}$ is more dominant in $S$. mutans identified from endocarditis samples. It has a defect at the glucose side chain in serotype-specific rhamnose-glucose polymers, which may be related to a higher incidence of detection in cardiovascular specimens, owing to reduced phagocytosis. These findings suggest that $S$. mutans is capable of surface interactions with different tissues and serotype $\mathrm{k} S$. mutans possibly has a higher level of virulence for systemic diseases [10].

Many surface proteins are attached to the bacterial cell wall by membrane-associated transpeptidases of the sortase family [11]. These enzymes cut the target proteins at a C-terminal cell wall sorting signal (CWSS), which is typically characterized by an LPxTG motif. This forms an acyl enzyme intermediate that covalently attaches the proteins to amino groups on peptidoglycan, resulting in incorporation into the cell envelope. Substrate proteins of sortases are initially expressed in a precursor with an
$\mathrm{N}$-terminal signal peptide and the C-terminal CWSS. The signal sequence directs the protein for translocation across the plasma membrane via the Sec secretion system' until the CWSS is reached. At this point, the protein is held in the membrane by a stretch of hydrophobic amino acids immediately downstream of the CWSS. The CWSS is then available for cleavage by the membrane-bound sortase, resulting in a protein that is exposed on the bacterial surface while securely embedded within the cell envelope.

Biofilm maturation stage is controlled by quorum sensing systems

Quorum sensing (QS) is a microbial cell-to-cell communication system designated for cell-density and/or population based gene regulation. Using a QS system, individual cells can produce and release small QS signaling molecules and detect the signal in the surrounding environment at same time. Several major types of QS systems have been identified and characterized, including $\mathrm{N}$-acyl-homoserine lactone (AHL) systems (from Gram negatives), 4-quinolone systems (from Gram negatives, hydrophobic signal), AgrD peptide systems (from Gram positives), and AI2/LuxS systems (both Gram negatives and Gram positives). Quorum sensing systems play critical roles in the maturing stage of biofilm formation and regulate cell differentiation and development of biofilm structures.

A breakdown of the cell-to-cell communication may keep cells in the planktonic state. It has been shown that a well-developed biofilm is more resistant to drug treatment than defective biofilms. Davies and colleagues demonstrated that a thin biofilm formed by the AHL quorum-sensing mutant lasl is more sensitive to treatment by antibiotics and sterilization solutions. This phenotype can be completely complemented by introducing a functional lasl or by adding the appropriate AHL [12].

\section{Clinical aspects of biofilms}

\section{Detection of biofilms is a practical concern}

From a clinical point of view, the first step towards a solution for biofilm-related infection is an early positive detection. The biofilm hypothesis predicts that biofilm cells are less sensitive to both nutritional stimulation and hostile attacks compared to planktonic cells. Additionally, biofilm fragments could be difficult to detect using traditional agar and culture based detection methods [3, 13]. Thus, evidence generated from direct microscopic examination and from molecular detections is preferred to verify a biofilm related condition. Although DNA and RNA based, species-specific, high-throughput detection 
methods are now recommended to screen clinical samples for the presence of pathogens, these technologies are neither affordable nor available in developing countries.

Currently, biofilm specific antigens and antibodies are being pursued for both diagnostic and therapeutic purposes. In 2002, Selan and her colleagues prepared surface slime polysaccharide adhesin (SSPA) that is highly expressed in biofilm cells, and established a SSPA-ELISA assay for anti-SSPA antibodies in clinical samples. The assay was used to monitor Staphylococcus biofilm formation in patients receiving synthetic vascular grafts. The particular clinical challenge is that Staphylococccal biofilms on the sutures would cause around $4 \%$ of the patient grafts to simply fail and rupture without any obvious warning symptoms. The SSPA-ELISA test would help clinicians to predict the occurrence of biofilm by comparing the test results obtained at different time points. The same technology was applied to periprosthetic joint infections [14].

Antigens from the methicillin-resistant Staphylococcus aureus (MRSA) cell wall have been shown to be immunogenic in vivo and up-regulated during in vitro biofilm growth. Purified and recombinant forms of biofilm-upregulated, cell wall-associated proteins were used for polyclonal immunoglobulin $\mathrm{G}$ (IgG) antibodies. These IgGs were utilized as imaging tools to localize areas of specific protein production within a biofilm [15]. These biofilm-specific or biofilm-enhanced antigens were applied as a therapeutic vaccine to treat MRSA caused conditions in a combination approach with vancomycin [16].

The Parsek-Singh biofilm criterion has been adopted by clinical microbiologists to track clinical biofilms. According to this criterion, a biofilm condition needs to match four conditions. (1) The pathogenic bacteria are surface associated or adherent to a substratum; (2) direct examination reveals bacteria in clusters are encased in a matrix of bacterial or host constituents; (3) the infection is localized; and, (4) the infection is resistant to antibiotic treatment despite the demonstrated susceptibility of planktonic bacteria. The guideline is a reflection of several key features of biofilms. With both DNA/RNA-based high-throughput detections and biofilm-specific antigens/ antibodies as our new tools, we are expecting simple and minimally invasive detection of biofilms in the clinical setting. A positive detection of a clinical biofilm would lead clinicians to employ therapeutic approaches appropriate for persistent infections associated with biofilms.

It should be pointed out that a detected biofilm is not always disastrous if it is not causing damage to surrounding tissues. As sessile bacteria provide a protected or isolated slow mode of growth, many biofilms are welltolerated in hosts. Dasgupta and colleagues examined more than 80 Tenchhoff catheters worn by continuous ambulatory peritoneal dialysis (CAPD) patients. Although thick biofilms were identified, the major clinical concern was acute peritonitis caused by the planktonic cells released from biofilms. The key clinical determining factor for peritonitis is host immune status and not the extent or species content of the biofilm [17].

What is our first clinical option for a detected problem? A recent review by Black and Costerton on chronic wound biofilms reasoned that as commercial topical agents and wound dressings currently in use are ineffective against the biofilm matrix, mechanical debridement appears to be essential in the eradication of a wound biofilm. Topical antimicrobial agents and antibiotics may be effective in the treatment of the wound bed after debridement in the prevention of biofilm reformation [18]. This removal/replacement plus medicine strategy is also applied for the treatment of periodontitis and most nosocomial infections.

\section{New tests are needed for drug selections for biofilms}

Biofilm formation is a main virulence determinant in many bacterial infections. It significantly increases bacterial resistance to antibiotics and innate host defenses. The drug-resistance strategy employed by sessile biofilm cells are different from the ones adopted by planktonic cells, such as activation of efflux pumps, acquisition of new enzymes and mutations of the drug targets. In general, the specific physiology of sessile bacteria and the physical and chemical barrier function of the extracellular biofilm matrix determine resistance to antimicrobials. As the sessile bacteria adopt a slow mode of growth, biofilms are relatively insensitive to bactericidal antibiotics. The effective drug concentration could be negatively affected by interactions of the biofilm matrix with antibiotics and antimicrobial peptides. These polymers may work by electrostatic repulsion and/or sequestration of antibacterial substances. Results indicated that biofilm cells may have a minimum inhibition concentration (MIC) that is 1000 times higher than planktonic cells.

How to select the best antibiotics to treat biofilmassociated conditions is a critical clinical challenge. Planktonic bacteria have been used in standard susceptibility tests to select the most appropriate antibiotic combinations to treat biofilm conditions, such as cystic fibrosis airway infections [19]. However, this approach has a key weak point: it does not consider the drug resistance impact posed by biofilms. Recently, biofilm susceptibility methods have been developed to address this concern [20-21]. Biofilm inhibitory concentrations or minimum biofilm eradication concentrations are higher 
than the corresponding MICs determined by standard planktonic methods. However, biofilm inhibitory concentrations and minimum biofilm eradication concentrations values vary greatly among the different biofilm susceptibility test methods, suggesting that the characteristics of the in vitro biofilms are conditional and strongly related to the laboratory system used to grow them [21]. This underscores the difficulty of selecting and developing biofilm inhibitors as well as compounds that potentiate the activity of antibiotics against biofilms. Clearly, it is important to test bacterial biofilms with clinically relevant antibiotic susceptibilities. Collective efforts from both clinicians and researchers are required to build standard biofilm inhibition and standard biofilm eradication assays. Biofilm-based strategies are needed to manage the patient's care and to develop new drug leads.

\section{Biofilms may cause inflammation}

Biofilms are protected from antibiotics and the body's immune system. For years, it was believed that leukocytes are not able to penetrate the biofilm. Leid and colleagues demonstrated that under simulated physiological conditions, leukocytes attach, penetrate, and produce cytokines in response to maturing and fully matured $S$. aureus biofilms [22]. Similar results were also observed from $P$. aeruginosa biofilms. Neutrophils that settle on biofilms appear to be unable to migrate away from the point of contact even though they are still capable of phagocytosis [23]. Neutrophil accumulation within biofilms may result in self-injury of the neutrophil by released oxidants which in turn compromises host defense mechanisms. Necrotic neutrophils can also serve as a biological matrix to facilitate $P$. aeruginosa biofilm formation [24].

Accumulated clinical evidence has indicated that biofilms associated with persistent infections can also cause chronic inflammation. Biofilm associated inflammations were identified from chronic wounds [25], cystic fibrosis [26], otitis media [27], osteomyelitis [28], prostatitis [29-30], and nosocomial infections. Anti-inflammation therapy is usually recommended for biofilms caused by systemic tissue infections.

As biofilm diseases are associated with both persistent infections and chronic inflammation, we anticipate future treatments to contain three active components, namely, antibiotics for both biofilm and planktonic cells, antiinflammatories for neutralizing the inflammation reactions generated from biofilms, and anti-biofilm compounds for the clearance of biofilms (3A remedies for biofilms). The next section of this review will discuss the current progress of anti-biofilm research.

\section{Research and development of anti-biofilm activities}

The road from biofilm hypothesis to biofilm therapy is promising, but long. After two decades of relentless effort and a quarter of a million publications, we are still waiting for the launch of the first anti-biofilm-based product. At this point, we are optimistic that the collective efforts in the field of anti-adhesion, quorum sensing disruption and species-specific killing will deliver new and practical biofilm based medicines and/or remedies.

\section{Sortase is a leading target for anti-adhesions}

At the adhesion stage of biofilm formation, adhesins expressed on the planktonic cell and/or biofilm fragments initiate surface/tissue specific attachment. As these surface interactions are critical for biofilm formation, hundreds of surface proteins identified from different microbial species are being studied as candidate adhesins for anti-adhesion compounds. The growing information on adhesins is creating opportunities and causing confusion at the same time. The surface protein repertoire enables multiple interactions with different host components and allows versatility when it comes to occupying adherence sites. The multiple adhesin-receptor interactions with various affinities represent some of the reasons for the difficulties that have been encountered in the characterization of adhesins. For example, single-gene knockouts may reveal very little about adherence mechanisms, and antibodies generated to specific surface proteins may have little or undetectable effects on adherence.

To make the best use of limited resources, researchers are trying to focus on the reactions/steps that are shared by most of the surface proteins, instead of working on individual adhesins. The sortase of Gram positives is such a candidate. For Gram positives, peptidoglycan inside the $20-100 \mathrm{~nm}$ thick cell wall is covalently and non-covalently decorated with teichoic acids, polysaccharides, and proteins. This dynamic surface structure is essential for different types of interactions between bacteria and their environments and for biofilm formation. Sortases are membrane enzymes catalyzing covalent anchoring of surface proteins to peptidoglycan $[11,31]$. Bacterial genome projects indicated that sortase is a common enzyme found in Gram positives with broad substrates. In S. aureus, around 20 candidate sortase substrates were identified from each sequenced strain, including protein $\mathrm{A}$ (Spa), fibronectin binding proteins (FnbpA, FnbpB), clumping factors (ClfA, ClfB), collagen adhesin (Can), heme binding proteins (IsdC, IsdB, IsdA) and other surface proteins [11]. The same is true for other Gram positives, such as S. epidermidis and $S$. mutans. Sortase A (srtA) mutants failed to display all 
surface proteins, while this phenotype could be rescued by a plasmid carrying the wild type gene. In animal disease models for sepsis, abscess, septic arthritis, and endocarditis, results demonstrated that the srtA mutant was less virulent than wild type strains [32-34]. Interestingly, two $S$. mutans clinical isolates contain sortase mutations in the srtA gene. S. mutans Ingbritt contains an 11-base-pair deletion in the $s r t A$ ORF that generates a premature stop codon [35], and $S$. mutans NG5 carries a missense mutation in the $\operatorname{srt} A$ gene that results in the production of a truncated, nonfunctional enzyme [36]. Both strains secrete PAc and are unable to adhere to hydroxyapatite and to aggregate in the presence of saliva. Based upon these studies, researchers are hopeful that sortase might serve as a good drug-target for antiadhesion activities. As sortase is a universal virulence factor for Gram positives, sortase inhibitors could have broad clinical applications.

Even before the identification of the enzyme, several compounds were recognized as sortase inhibitors, including methane-thiosulfonate and mercurial p-hydroxymercuribenzoic acid. These compounds function by blocking the Cys184 in the active pocket of sortase A. Although these compounds are useful for illustrating the action model of the enzyme, they are of limited therapeutic value due to their general toxicities. Natural compound libraries were screened for candidate inhibitors. Compounds isolated from Cocullus trilobus and Coptis chinensis exhibited lower MICs than p-hydroxymercuriabenzoic acid and were able to block the adherence of bacteria to fibronectin coated surfaces [37-38]. Libraries of small compounds have also been screened, and (Z)-diarylacrylonitriles were identified as potential leading compounds. Another strategy was to identify irreversible sortase inhibitors by screening modified versions of LPXTG, the common sortase binding site on substrates. Candidate inhibitors, such as diazoketone LPAT (LPAT-COCHN ${ }_{2}$ ), chloromethyl ketone LPAT (LPAT$\mathrm{COCH}_{2} \mathrm{Cl}$ ), were isolated for further studies [38]. The key technical issues for sortase-based anti-adhesions, such as assay design and animal models, are discussed in a review by Maresso and Schneewind.

\section{Biofilm formation can be blocked by quorum sensing inhibitors (QSI)}

At the maturation stage of biofilm formation, bacteria use cell-to-cell communication systems to regulate the expressions of the genes required and/or actively involved in the biofilm formation. A breakdown of the cell-to-cell communication may keep cells in the planktonic state. QS is a microbial cell-to-cell communication system designated for cell-density and/or population based gene regulation. Using a QS system, individual cells can produce and release small QS signaling molecules and detect signals in the surrounding environment at same time. Since the discovery of QS in the 1960s [39], several major types of QS systems have been identified and characterized, including N-acyl-homoserine lactone (AHL) systems (from Gram negatives), 4-quinolone systems (from Gram negatives, hydrophobic signal), AgrD peptide systems (from Gram positives), AI2/LuxS systems (both Gram negatives and Gram positives), and farnesol systems (from fungi). As accumulated publications imply that QS systems are actively involved in controlling biofilm formation and infections, researchers are focusing their efforts on quorum sensing inhibitors (QSI) for potential new therapeutics [40].

Among a large number of reported QSIs, furanones and RNA III inhibiting peptide (RIP) are two classes of leading candidates. Furanones have been extensively studied for their activities and for QS inhibitory mechanisms. Several lines of evidence indicate that the furanones may act on both AHL systems and AI2 systems. Some furanones devoid of growth inhibitory effects can increase the susceptibility of biofilms to antibiotics and detergents [41]. In animal disease models, furanones could facilitate the host immune systems to clear infections [42]. Antibodies against AHLs have also been generated after conjugating AHL with carrier proteins [43]. The antibodies might have promising applications in disease control.

A linear Agr QS inhibitor known as RNA III inhibiting peptide in its amide form (RIP, YSPWTNF-NH ${ }_{2}$ ) has been shown to repress virulence, biofilm formation, and antibiotic resistance in staphylococci [44-45]. A simple mechanism of RIP function is proposed. As the cells multiply, the QS signal RAP accumulates in the supernatant, binds to its receptor, and induces the phosphorylation of TRAP. $a g r$ is then activated at the mid exponential phase of growth and RNA II is produced and AgrA-D are made. This leads to the production of RNA III, which up regulates the production of toxins at the post-exponential phase of growth. In the presence of RIP, TRAP is not phosphorylated, agr is not activated, and toxins are not produced. Despite promising results obtained from several animal models, the clinical application of RIP and its synthetic analogues is still in its early stage. The in vivo product stability and toxicity of the peptide drug are two potential concerns.

Selectively targeting antimicrobial peptides (STAMPS) is a new option for species-specific control of biofilms

Treatment of persistent infections caused by pathogens sitting in a functional biofilm community, such as 
dental caries, is a clinical challenge. Conventional broadspectrum antimicrobial treatment attacks a subpopulation of the biofilm, and may cause new problems by eliminating commensals. To build a pathogen-free biofilm, species-specific vaccines [46], anti-adhesins, (discussed above) and replacement therapies [47] have been explored. Recently, a new targeted approach named selectively targeted antimicrobial peptides (STAMPs) is emerging as a promising new strategy for species-specific biofilm control.

In short, STAMP is a modified recombinant version (or third generation) of antimicrobial peptides (AMPs). AMPs are widespread in nature and play an important role as part of innate immunity. In general, AMPs are fairly large molecules that carry a net positive charge and contain around 50\% hydrophobic residues. Their mode of action involves binding to negatively-charged structural molecules on the microbial membrane. AMPs have a broad spectrum of antimicrobial activity and development of resistance is rare. Unfortunately, AMPs are difficult and expensive to produce in large quantities and are usually sensitive to protease digestion. Modifications of AMPs have resulted in the development of synthetic antimicrobial peptides, also called SAMPs (second generation of AMPs). SAMPs mimic the effect of AMPs, but have improved pharmacokinetic properties. Despite these improvements, SAMPs are still broad spectrum. This problem was solved by STAMPs.

STAMPs represent a novel strategy initially developed by Shi and his colleagues to remove pathogens from multi-species communities and/or specific clinical settings [48]. A typical STAMP contains a species-specific binding domain to facilitate the delivery of a conjoined antimicrobial peptide. The targeting peptide can be identified via screening peptide libraries or rational design. Targeting peptides specifically binding to $S$. mutans is derived from bacterial pheromones such as CSP (SGSLSTFFRLFNRSFTQALGK), and a targeting peptide specific for $P$. aeruginosa is derived from KKHRKHRKHRKH. A peptide of 2 to 20 amino acids is used to connect a targeting peptide to an antimicrobial peptide without interfering or reducing the activity. The technology has been applied to remove both Gram-positive strains [48-50] and Gram-negative strains [48-49]. Recently, a STAMP with two species-targeting domains was used to treat both $S$. mutans and Pseudomonas in a multi-species culture [49]. In an in vitro dynamic biofilm model, Li and colleagues demonstrated that after selective elimination of $S$. mutans from the existing biofilm with a STAMP, the treated biofilms are resistant to the recolonization of newly added $S$. mutans [50]. Although the STAMP technology may face the same challenges as
RI, AMPs and other peptide based new drug candidates, STAMPs have a strong potential for novel therapeutics that may selectively eliminate pathogens, while preserving the benefits associated with a healthy normal flora.

\section{Summary}

Biofim formation is a two-stage biological process controlled by surface adhesins and cell-to-cell communication pathways. Aggregated bacterial cells protected and/or coated by extracellular matrix are insensitive to both nutritional stimulation and hostile attacks. In the human body, biofilms may trigger persistent infections with chronic inflammation. After a positive detection of biofilm related medical conditions, both surgical removal/ replacement and medicinal treatment should be considered. Ideally, an effective remedy for biofilm associated conditions should contain antibiotics, anti-inflammatories, and anti-biofilm activities (3A remedies).

The road from molecular mechanisms of biofilm formation to anti-biofilm products is promising, but long. Non-invasive and/or minimally invasive detection methods and standard biofilm assays that mimic clinical conditions are opening the door for new, biofilmoriented solutions. A large number of biofilm inhibitors are currently under comprehensive investigation. If clinicians are made more aware of the importance of bacterial biofilm formation and their associated diseases, more translational research will be designed and new therapeutic approaches may be developed.

\section{Acknowledgement}

The authors are grateful to Drs. Di Qu, Qian Gao, and Justin Merritt for their helpful discussions.

\section{References}

1 Singh PK, Schaefer AL, Parsek MR, et al. Quorum-sensing signals indicate that cystic fibrosis lungs are infected with bacterial biofilms. Nature 2000; 407: 762-764.

2 Anderson GG, Dodson KW, Hooton TM, Hultgren SJ. Intracellular bacterial communities of uropathogenic Escherichia coli in urinary tract pathogenesis. Trends Microbiol 2004; 12: 424-430.

3 Ojha AK, Baughn AD, Sambandan D, et al. Growth of Mycobacterium tuberculosis biofilms containing free mycolic acids and harbouring drug-tolerant bacteria. Mol Microbiol 2008; 69: 164-174.

4 Costerton JW, Veeh R, Shirtliff M, et al. The application of biofilm science to the study and control of chronic bacterial 
infections. J Clin Invest 2003; 112: 1466-1477.

5 Costerone JW, Stewart PS, Greenberg EP. Bacterial biofilms: a common cause of persistent infections. Science 1999; 284: 1318-1322.

6 Balaban NQ, Merrin J, Chait R, Kowalik L, Leibler S. Bacterial persistence as a phenotypic switch. Science 2004; 305: $1622-1625$.

7 Tojo M, Yamashita N, Goldmann DA, Pier GB. Isolation and characterization of a capsular polysaccharide adhesin from Staphylococcus epidermidis. J Infect Dis 1988; 157: 713-722.

8 Zhang YQ, Ren SX, Li HL, et al. Genome-based analysis of virulence genes in a non-biofilm-forming Staphylococcus epidermidis strain (ATCC 12228). Mol Microbiol 2003; 49: 1577-1593.

9 Nobbs AH, Lamount RJ, Jenkinson H. Streptococcus adherence and colonization. Microbiol Mol Biol Rev 2009; 73: 407-505.

10 Nakano K, Nomura R, Shimizu N, et al. Development of a PCR method for rapid identification of new Streptococcus mutans serotype k strains. J Clin Microbiol 2004; 42: 49254930.

11 Marraffini LA, Dedent AC, Schneewind O. Sortases and the art of anchoring proteins to the envelopes of Gram-positive bacteria. Microbiol Mol Biol Rev 2006; 70: 192-221.

12 Davies DG, Parsek MR, Pearson JP, et al. The involvement of cell-to-cell signals in the development of a bacterial biofilm. Science 1998; 280: 295-298.

13 Veeh RH, Shirtliff ME, Petik JR, et al. Detection of Staphylococcus aureus biofilm on tampons and menses components. J Infect Dis 2003; 188: 519-530.

14 Artini M, Romano C, Manzoli L, et al. Staphylococcal IgM ELISA for the detection of periprosthetic joint infections. J Clin Microbiol 2011; 49: 423-425.

15 Brady RA, Leid JG, Kofonow J, Costerton JW, Shirtliff ME. Immunoglobulins to surface-associated biofilm immunogens provide a novel means of visualization of methicillin-resistant Staphylococcus aureus biofilms. Appl Environ Microbiol 2007; 73: 6612-6619.

16 Brady RA, O'May GA, Leid JG, Costerton JW, Shirtliff ME. Resolution of Staphylococcus aureus biofilm infection using vaccination and antibiotic treatment. Infect Immun 2011; [Epub ahead of print].

17 Dasgupta MK, Bettcher KB, Ulan RA, et al. Relationship of adherent bacterial biofilms to periotonitis in chronic ambulatory periotoneal dialysis. Periot Dial Bull 1987; 7: 168173.

18 Black CE, Costerton JW. Current concepts regarding the effect of wound microbial ecology and biofilms on wound healing. Surg Clin North Am 2010; 90: 1147-1160.

19 Saiman L, Burns JL, Whittier S, et al. Evaluation of reference dilution test methods for antimicrobial susceptibility testing of Pseudomonas aeruginosa strains isolated from patients with cystic fibrosis. J Clin Microbiol 1999; 37: 2987-2991.

20 Ceri H, Olson ME, Stremick C, et al. The Calgary biofilm device: new technology for rapid determination of antibiotic susceptibilities of bacterial biofilms. J Clin Microbiol 1999; 37: 1771-1776.

21 Moskowitz SM, Foster LM, Emerson J, Burns JL. Clinically feasible biofilm susceptibility assay for isolates of Pseudomonas aeruginosa from patients with cystic fibrosis. $J$ Clin Microbiol 2004; 42: 1915-1922.

22 Leid JG, Shirtliff ME, Costerton JW, Stoodley P. Human leukocytes adhere to, penetrate, and respond to Staphylococcus aureus biofilms. Infect Immun 2002; 70: 6339-6345.

23 Jesaitis AJ, Franklin MJ, Berglund D, et al. Compromised host defense on Pseudomonas aeruginosa biofilms: characterization of neutrophil and biofilm interactions. J Immunol 2003; 171: 4329-4339.

24 Walker TS, Tomlin KL, Worthen GS, et al. Enhanced Pseudomonas aeruginosa biofilm development mediated by human neutrophils. Infect Immun 2005; 73: 3693-3701.

25 Wolcott RD, Rhoads DD, Dowd SE. Biofilms and chronic wound inflammation. $J$ Wound Care 2008; 17: 333-341.

26 Ciofu O, Riis B, Pressler T, Poulsen HE, Høiby N. Occurrence of hypermutable Pseudomonas aeruginosa in cystic fibrosis patients is associated with the oxidative stress caused by chronic lung inflammation. Antimicrob Agents Chemother 2005; 49: 2276-2282.

27 Rayner MG, Zhang Y, Gorry MC, et al. Evidences of bacterial metabolic activities in culture-negative otitis media with effusion. J Am Med Assoc 1998; 279: 296-299.

28 Wagner C, Kondella K, Bernschneider $\mathrm{T}$, et al. Posttraumatic osteomyelitis: analysis of inflammatory cells recruited into the site of infection. Shock 2003; 20: 503-510.

29 Nickel JC, Costerton JW, McLean JC, Olson M. Baterial biofilms: influence on the pathogenesis, diagnosis and treatment of urinary-tract infections. J Antimicrob Chemother 1994; 33: 31-41.

30 Mazzoli S. Biofilms in chronic bacterial prostatitis (NIH- II ) and in prostatic calcifications. FEMS Immunol Med Microbiol 2010; 59: 337-344.

31 Mazmanian SK, Liu G, Ton-That H, Schneewind O. Staphylococcus aureus sortase, an enzyme that anchors surface proteins to the cell wall. Science 1999; 285: 760 763.

32 Jonsson IM, Mazmanian SK, Schneewind O, et al. The role of Staphylococcus aureus sortase A and sortase B in murine arthritis. Microbes Infect 2003; 5: 775-780.

33 Weiss WJ, Lenoy E, Murphy T, et al. Effect of $s r t A$ and $s r t B$ gene expression on the virulence of Staphylococcus aureus in animal models of infection. J Antimicrob Chemother 2004; 53: 480-486.

34 Gianfaldoni C, Maccari S, Pancotto L, et al. Sortase A 
confers protection against Streptococcus pneumoniae in mice. Infect Immun 2009; 77: 2957-2961.

35 Igarashi T. Deletion in sortase gene of Streptococcus mutans Ingbritt. Oral Microbiol Immunol 2004; 19: 210-213.

36 Lee, SF, McGavin MK. Identification of a point mutation resulting in loss of cell wall anchoring activity of SrtA of Streptococcus mutans NG5. Infect Immun 2004; 72: 4314 4317.

37 Kim SW, Chang IM, Oh KB. Inhibition of the bacterial surface protein anchoring transpeptidase sortase by medicinal plants. Biosci Biotechnol Biochem 2002; 66: 2751-2754.

38 Maresso AW, Schneewind O. Sortase as a target of antiinfective therapy. Pharmacol Rev 2008; 60: 128-141.

39 Tomasz A. Control of the competent state in Pneumococcus by a hormone-like cell product: an example for a new type of regulatory mechanisms in bacteria. Nature 1965; 208: $155-159$.

40 Asad S, Opal SM. Bench-to-bedside review: quorum sensing and the role of cell-to-cell communication during invasive bacterial infection. Crit Care 2008; 12: 236-247.

41 Pan JC, Ren DC. Quorum sensing inhibitors: a patent review. Expert Opin Ther Patents 2009; 19: 1581-1601.

$42 \mathrm{Wu} \mathrm{H}$, Song Z, Hentzer M, et al. Synthetic furanones inhibit quorum-sensing and enhance bacterial clearance in Pseudomonas aeruginosa lung infection in mice. $J$ Antimicrob Chemother 2004; 53: 1054-1061.

43 Kaufmann GF, Sartorio R, Lee SH, et al. Antibody interference of $\mathrm{N}$-acyl-homoserine lactone-mediated bacteria quorum sensing. J Am Chem Soc 2006; 128: 2802-2803.

44 Balaban N, Giacometti A, Girioni O, et al. Use the quorumsensing inhibitor RNAIII-inhibiting peptide to prevent biofilm formation in vivo by drug resistant Staphylococcus epidermidis. J Infect Dis 2003; 187: 625-630.

45 Balaban N, Cirioni O, Giacometti A, et al. Treatment of Staphylococcus aureus biofilm infection by the quorumsensing inhibitor RIP. Antimicrob Agents Chemother 2007; 51: 2226-2229.

46 Li LN, Guo LH, Lux R, et al. Targeted antimicrobial therapy against Streptococcus mutans establishes protective noncariogenic oral biofilms and reduces subsequent infection. Int J Oral Sci 2010; 2: 66-73.

47 Hillman JD, Brooks TA, Michalek SM, et al. Construction and characterization of an effector strain of Streptococcus mutans for replacement therapy of dental caries. Infect Immun 2000; 68: 543-549.

48 Eckert R, Qi F, Yarbrough DK, et al. Adding selectivity to antimicrobial peptides: rational design of a multidomain peptide against Pseudomonas spp.. Antimicrob Agents Chemother 2006; 50: 1480-1488.

49 He J, Anderson MH, Shi W, Eckert R. Design and activity of a 'dual-targeted' antimicrobial peptide. Int $J$ Antimicrob Agents 2009; 33: 532-537.

50 Li YH, Huang S, Du M, et al. Immunogenic characterization and protection against Streptococcus mutans infection induced by intranasal DNA prime-protein boost immunization. Vaccine 2010; 28: 5370-5376. 